\title{
\begin{tabular}{l|l|l|l|l}
\hline M & R & S & Internet Journal of & Nitride Semiconductor Research \\
\hline
\end{tabular}
}

Volume 2, Article 2

\section{Interfacial Reactions and Electrical Properties of Ti/n-GaN Contacts}

\author{
Holger Cordes, Y. A. Chang \\ Department of Materials Science and Engineering, University of Wisconsin, Madison \\ This article was received on December 17, 1996 and accepted on January 22, 1997.
}

\begin{abstract}
The phase equilibria in the ternary Ti-Ga-N have been investigated. Interfacial reactions in Ti/GaN contacts have been studied by diffusion couple experiments. The ternary phase $\mathrm{Ti}_{2} \mathrm{GaN}$ was confirmed by x-ray diffraction in bulk samples as well as in massive Ti/GaN diffusion couples and annealed Ti thin films on $\mathrm{GaN}$. The diffusion path in samples, annealed at $850^{\circ} \mathrm{C}$ in $\mathrm{Ar}$ gas, is $\mathrm{GaN} / \mathrm{TiN} / \mathrm{Ti}_{2} \mathrm{GaN} / \mathrm{Ti}_{3} \mathrm{Ga} / \mathrm{Ti}$. A planar TiN layer forms in direct contact to $\mathrm{GaN}$ and governs the electrical properties of annealed Ti/GaN contacts. Thin film contacts were fabricated by sputtering Ti on MOVPE grown $n-G a N\left(5 \times 10^{17} \mathrm{~cm}^{-3}\right)$ and subsequent rapid thermal annealing in an Argon atmosphere. Initially non-linear current-voltage characteristics become ohmic after annealing and a specific contact resistance of approximately $10^{-2} \Omega \mathrm{cm}^{2}$, measured with the circular transmission line method, was found after annealing at $900^{\circ} \mathrm{C}$ for $1 \mathrm{~min}$.
\end{abstract}

\section{Introduction}

With the recent success in the development of gallium nitride based devices, such as blue LEDs and laser diodes, the fabrication of reliable ohmic and Schottky contacts to GaN has become a more important issue. Making reliable ohmic contacts to wide band gap semiconductors with sufficiently low contact resistance is usually a difficult task. Although a Schottky barrier height of $0.58 \mathrm{eV}$ was measured for Ti on n-GaN [1], several ohmic contact structures with Ti have been used [2] [3] [4]. The contact with the currently lowest observed specific contact resistance $\left(8 \times 10^{-6}\right.$ $\Omega \mathrm{cm}^{2}$ ) on $\mathrm{n}-\mathrm{GaN}$ is fabricated by deposition of a Ti/Al bi-layer followed by an Rapid Temperature Annealing (RTA) at $900^{\circ} \mathrm{C}$ for $30 \mathrm{sec}$ [2]. The specific contact resistance could be improved even further by the use of pre-deposition reactive ion etching (RIE) and the introduction of an additional $\mathrm{Ni} / \mathrm{Au}$ capping layer leading to a rather complex contact structure [5]. Both contacts are not understood in detail yet and explanations are speculative. As Lin at al. [2] pointed out, an analysis of the interface zone of the contact is hampered by the absence of the quaternary Ga-N-Ti-Al phase diagram. In a recent survey of transition metal-Ga-N phase equilibria a tieline between TiN and GaN was predicted in the ternary Ti-Ga-N by approximate calculations; however, a ternary phase was not included due to the lack of thermodynamic data [6]. These data as well as the knowledge of the interface morphology between contact and semiconductor are essential for the development of a reliable, thermally stable contact technology.

The purpose of this study is:

- to provide the present fundamental data about the phase equilibria in the ternary Ti-Ga-N phase diagram

- to study the Ti/GaN interface by means of diffusion couple experiments

- to fabricate and characterize electrical Ti/GaN contacts and correlate their properties with the observed interface behavior.

These data can possibly lead to a better understanding of the currently used contact structures and provide a starting point for the investigation of the more complicated phase relations in the quaternary Ti-Al-Ga-N system. 


\section{Phase equilibria in the Ga-N-Ti system}

\subsection{Approximate Calculation and Review of the Literature}

In the binary Ti-Ga system up to eight different phases were reported but some of the observed structures were not established as equilibrium phases [7]. In particular the region with more than 35 at.\% Ga, remains uncertain. The only phase diagram in the whole composition range, given by Pötschke [8] is partly questionable due to oxide contamination of the samples. All intermediate phases, given by Murray [7] were accepted for the approximate calculation, although the existence of some phases remains uncertain.

In the Ti-N binary two phases TiN and $\mathrm{Ti}_{2} \mathrm{~N}$ exist and in the Ga-N binary only GaN. Very few data were available for the Ga-N-Ti ternary system. A ternary phase $\mathrm{Ti}_{2} \mathrm{GaN}$ was reported [9]; however, no confirmation and no thermodynamic data were available for this phase.

An approximate calculation of the phase equilibria in the Ga-N-Ti ternary system was performed by means of the TerQuat program [10]. In this program all phases are assumed to be line compounds. The Gibbs energy for all phases was either taken from the literature, or approximated by the enthalpy of formation, as predicted by the Miedema model [11]. In the Ti-N binary Gibbs energies of $-154.6 \mathrm{~kJ} / \mathrm{g}$-atom (TiN), respectively -104 kJ/g-atom (Ti2N), were taken from Kubaschewski's compilation [12] and for $\mathrm{GaN}$, a value of $-70.7 \mathrm{~kJ} / \mathrm{g}$-atom [12] was used in the calculation.

The Gibbs energies for the accepted phases in the Ti-Ga binary are TiGaz (-31kJ/g-atom), TiGa 2 (-41 kJ/g-atom), Ti ${ }_{3} \mathrm{Ga}_{5}\left(-45 \mathrm{~kJ} / \mathrm{g}\right.$-atom), $\mathrm{Ti}_{2} \mathrm{Ga}_{3}\left(-47 \mathrm{~kJ} / \mathrm{g}\right.$-atom), TiGa (-51kJ/g-atom), $\mathrm{Ti}_{5} \mathrm{Ga}_{4}\left(-48 \mathrm{~kJ} / \mathrm{g}\right.$-atom), $\mathrm{Ti}_{5} \mathrm{Ga}_{3}(-46 \mathrm{~kJ} / \mathrm{g}$-atom) Ti ${ }_{2} \mathrm{Ga}\left(-44 \mathrm{~kJ} / \mathrm{g}\right.$-atom) and $\mathrm{Ti}_{3} \mathrm{Ga}(-35 \mathrm{~kJ} / \mathrm{g}$-atom).

Using the above data for the calculation, a minimum Gibbs energy of formation of $-103 \mathrm{~kJ} / \mathrm{g}$-atom is required for $\mathrm{Ti}$ ${ }_{2} \mathrm{GaN}$ to be stable at room temperature. The isothermal section of Figure 1 was calculated using a value of -110 $\mathrm{kJ} / \mathrm{g}$-atom.

According to the approximate calculation the connection line between $\mathrm{GaN}$ and $\mathrm{Ti}$ is crossed by several tielines, hence an interface reaction in Ti/GaN diffusion couples is almost certain. It can also be seen that a three phase field GaN-Ga-TiN forms, which leaves TiN or Ga as possible phases in contact to GaN. However, if the estimated Gibbs energy for $\mathrm{Ti}_{2} \mathrm{GaN}$ is lowered below $-115 \mathrm{~kJ} / \mathrm{g}$-atom, a GaN-Ti2 $\mathrm{GaN}$ tieline would form, the most likely reaction mechanism in diffusion couples would be altered and $\mathrm{Ti}_{2} \mathrm{GaN}$ would be in direct contact with $\mathrm{GaN}$.

This means that the existing thermodynamic data in the system are not sufficient to predict the phase equilibria without doubt; however, few selected samples in the ternary system or the experimental observation of a diffusion path are sufficient to verify the approximate calculation of phase equilibria and in turn give a range for the Gibbs energy value of the ternary compound.

\subsection{Experimental phase diagram study}

The experiments were focused on the verification of the ternary $\mathrm{Ti}_{2} \mathrm{GaN}$ phase, since no report other than from Jeitschko [9] was available. Starting materials were TiN, Ga and Ti with $3 \mathrm{~N}$ purities. The nominal composition was weighted, thoroughly mixed and cold pressed to a pellet. The sample was then sealed in an evacuated silica tube and annealed at $850^{\circ} \mathrm{C}$ for 160 hours. After quenching in water the sample was studied by XRD with CuKo radiation.

The quenched sample was visibly two-phase. Most of it consisted of a dark gray phase, which was separated from the gold colored TiN with tweezers. The dark phase was powdered and resulted in an almost single phase XRD-spectrum (Figure 2) which was almost identical with the calculated spectrum from literature data [13] (using [14]). Only a small peak could be attributed to the strongest TiN refraction. This result confirms that TígaN is a stable phase at $850^{\circ} \mathrm{C}$; however, additional experiments or the identification of a diffusion path are necessary to obtain the tielines in the system.

\section{Diffusion studies}




\subsection{Experimental}

In order to investigate the reaction zone between $\mathrm{Ti}$ and GaN several Ti/GaN diffusion couples were prepared. Starting materials for massive diffusion couples were thick layers of GaN $(80 \mu \mathrm{m})$, grown by Halide Vapor Phase Epitaxy (HVPE) on (0001) sapphire [15] and a Ti foil which was cleaned in $\mathrm{HF}: \mathrm{H}_{2} \mathrm{O}$ (1:1) solution before use. Both,

Ti foil and GaN substrate, were placed above each other and were cold pressed with Ti powder to a pellet of $5 \mathrm{~mm}$ diameter. The samples were sealed in silica under several mbar argon atmosphere and annealed at temperatures between $700^{\circ} \mathrm{C}$ and $850^{\circ} \mathrm{C}$. After quenching, the pellets were embedded in epoxy and a cross section through the reaction zone was prepared metallographically by grinding and polishing. The polished sections were examined by optical microscopy as well as by scanning electron microscope (SEM) with energy dispersive X-ray spectroscopy (EDX). From a linescan across the interface the diffusion path could not be identified unambiguously because a quantitative determination of the $\mathrm{N}$ content was not feasible.

For this reason a second preparation method for diffusion couples was applied to determine the phase structure in the interface zone by XRD and to verify the suspected $\mathrm{Ti}_{2} \mathrm{GaN}$ phase in the sample. A thick Ti layer ( $\left.\approx 400 \mathrm{~nm}\right)$ was sputter deposited onto the GaN substrate and the sample was subsequently annealed in a sealed silica tube at $850^{\circ} \mathrm{C}$ for 24 hours to ensure a complete reaction of the film.

An XRD-spectrum of the thin film was measured in glancing angle X-ray diffraction mode with a $10^{\circ}$ angle of incidence.

\subsection{Results}

A cross sectional view of the reaction zone of a sample annealed at $850^{\circ} \mathrm{C}$ for 2 days is shown in Figure $3 a$. The corresponding EDX line scan in Figure $3 b$ gives a qualitative measure of the $\mathrm{Ga}$ and Ti content across the interface. A basically planar layered structure can be observed with a thin dark layer in direct contact to GaN. From the little drop in Ga content at the interface and from the darker color in the Back Scattered Electron image, which corresponds to lower density of the phase, it is clear that a thin TiN layer forms at the interface. A more significant drop in Ga content was not observed because the excitation volume of the electron beam and the TiN layer thickness have comparable size. The subsequent, much thicker, layer is most likely $\mathrm{Ti}_{2} \mathrm{GaN}$ followed by a $\mathrm{Ti}_{3} \mathrm{Ga}$ layer close to the $\mathrm{Ti}$. Within the $\mathrm{Ti}$ ${ }_{3}$ Ga layer voids developed in all samples.

The $\mathrm{Ti}_{2} \mathrm{GaN}$ phase was also verified by XRD in the completely reacted thin film sample, as shown in Figure 4. In addition, a strong GaN peak and the strongest peaks of the $\mathrm{Ti}_{3} \mathrm{Ga}$ and $\mathrm{TiN}$ phases were identified in this sample. However, not all peaks could be attributed to possible reaction products. They probably correspond to phases caused by oxygen contamination.

\section{Electrical characterization of $\mathrm{Ti} / \mathrm{GaN}$ contacts}

\subsection{Experimental}

Epitaxially grown $n$-GaN layers of approximately $2 \mu \mathrm{m}$ thickness on (0001) sapphire with AIN bufferlayers were used as substrate material. The growth was carried out in a horizontal MOVPE reactor with Trimethylgallium, Trimethylaluminium and ammonia as reactants. A X-ray double crystal scan of the 002 GaN peak revealed a full width half maximum of 380 arcsec. Unintentionally doped layers had typically a carrier density of $2-5 \times 10^{17} \mathrm{~cm}^{-3}$ and a Hall mobility around $300 \mathrm{~cm}^{2} / \mathrm{V} \mathrm{sec}$ Before deposition of the contacts the surface was cleaned by degreasing in ultrasonically agitated trichloroethylene, acetone and methanol for 5 minutes each. In a second step the GaN was etched in boiling $\mathrm{NaOH}$ solution for 5 min and subsequently rinsed in deionized (DI) water.

Photo lithography with lift-off was applied to fabricate the contact pattern. Before metal deposition the contact holes were etched again using various solutions, that were reported in the literature. For the contact measurements, presented in this paper, a $\mathrm{HCl}: \mathrm{H}_{2} \mathrm{O}(1: 1)$ solution was used for $5 \mathrm{~min}$, followed by aDl- $\mathrm{H}_{2} \mathrm{O}$ rinse. Afterwards the substrates were blown dry with nitrogen and immediately transferred to a sputter chamber with a base pressure of $5 \times 10^{-7}$ mbar.

Polycrystalline Ti-contacts were sputter-deposited with a typical thickness of $100 \mathrm{~nm}$ at a sputter rate of approximately $0.1 \mathrm{~nm} / \mathrm{sec}$ and with an accelerating voltage of $290 \mathrm{~V}$. Several ring patterns of different size were 
deposited on the substrate for circular transmission line measurements [16]. The inner contact dot had a radius between 30 and $50 \mu \mathrm{m}$.

Besides these structures to measure the specific contact resistance of the annealed contacts, part of the substrate was prepared as a large area Al contact in order to measure the barrier height of the as-deposited Ti contacts. Al forms an ohmic contact even in the as-deposited state, but degrades rapidly upon annealing [2]. One half of the patterned substrate was covered by a metal foil and an Al layer was sputter deposited on top of some Ti structures, in order to form a large ohmic contact area. After annealing at higher temperature the current flow occurs mainly across the Al/Ti structures. The current-voltage (I-V) characteristics were measured between the inner contact dots and the large ohmic contact area. Electrical characterization was performed using a Source Measure Unit (Keithley 236) and copper beryllium needle probes. Various annealing procedures between $400^{\circ} \mathrm{C}$ and $900^{\circ} \mathrm{C}$ for 60 sec were used in a AG Associates Mini Pulse RTA-furnace under a purified Argon atmosphere. The heating rate for all experiments was $30 \% \mathrm{sec}$.

\subsection{Results}

Current-voltage (I-V) curves of a Ti/GaN contact, annealed at different temperatures, are shown inFigure 5 . In the as-deposited state the contacts were always nonlinear. The determination of the barrier height using the I-V curve was unreliable, since the measured ideality factor had typically a value of $n=3$. The corresponding barrier height was $\phi_{\mathrm{B}}=0.9 \mathrm{eV}$, calculated from the saturation current density with the theoretical value for the effective Richardson constant of $24 \mathrm{~A} \mathrm{~cm}^{-2} \mathrm{~K}^{-2}[17]$.

Upon annealing at $400^{\circ} \mathrm{C}$ the contacts became ohmic and the I-V curves remained almost unchanged up to a $900^{\circ} \mathrm{C}$, some of the corresponding I-V curves in Figure 5 are omitted for clarity. The large area Al contact showed considerable degradation upon annealing, however the reacted Ti/Al structure ensured the current flow through the ohmic contact.

After annealing at $950^{\circ} \mathrm{C}$ the I-V curve showed considerable degradation, but remained linear although the determination of the specific contact resistance was no longer possible with the Circular Transmission Line method due to an increased sheet resistance of the contact. The specific contact resistance for annealed contacts was in the range between $10^{-1}$ and $10^{-2} \Omega \mathrm{cm}^{2}$, as shown in Figure 6 .

Various pre-deposition substrate treatments were tried in order to improve contact performance but no other wet etch solution, that was used, showed a significant improvement. However, it was noticed that lower specific contact resistance measurements were generally obtained on substrates that had experienced some heat treatment prior to contact deposition.

\section{Discussion}

From the combination of both diffusion couple experiments a $\mathrm{GaN} / \mathrm{TiN} / \mathrm{Ti}_{2} \mathrm{GaN} / \mathrm{Ti}_{3} \mathrm{Ga} / \mathrm{Ti}$ diffusion path could be identified at reacted Ti/GaN interfaces. The observed diffusion path is superimposed on the calculated isothermal section (Figure 1) at room temperature. With the assumption that the phase equilibria remain in principal unchanged between room temperature and $850^{\circ} \mathrm{C}$, a $\Delta \mathrm{G}_{\mathrm{f}}$ value between -103 and $-115 \mathrm{~kJ} / \mathrm{g}$-atom can be estimated for the Ti ${ }_{2}$ GaN phase.

The reaction mechanism in diffusion couples is not clear yet, because marker experiments were not performed, but it seems likely that the reaction front moves mainly towards the GaN layer, because the observed gaps always developed on the Ti-side of the diffusion couple.

The electrical properties can indirectly be correlated to the observed interface morphology. The measurements show that TiN forms an ohmic contact to $n-G a N$ which is thermodynamically stable up to high annealing temperatures. From the electrical characterization a reaction between the Ti-film and the substrate seems to occur at temperatures as low as $400^{\circ} \mathrm{C}$, although no reaction could be detected in massive diffusion couples that were annealed at temperatures below $700^{\circ} \mathrm{C}$. However, as expected from the layered interface structure in diffusion couples, the I-V curves remain almost unchanged upon annealing at temperatures up to $900^{\circ} \mathrm{C}$. This is in an indication that $\mathrm{TiN}$ is the only phase in contact with GaN and therefore governs the electrical properties of the alloyed contact.

The specific contact resistance of TiN contacts in this study was considerable higher than the values measured for annealed Ti/Al contacts [2] [5]. The substrates, used in this study, had a considerably lower carrier density which is 
the main cause of the observed high specific contact resistance. Guo et al. [3] had measured Ti/Ag specific contact resistance as a function of substrate carrier density and observed a rapid decrease when the carrier density becomes larger than $1 \times 10^{18} \mathrm{~cm}^{-3}[3]$. For similarly doped substrates their results are well in accordance with this study.

The transport mechanism in Ti-based ohmic contacts can be explained by different models. Originally a strong dependence of the barrier height on the metal work function was anticipated for GaN due to its ionic nature [18]. However, if one compares the work functions of gallium nitride (4.1 eV [19]) and polycrystalline Ti $4.33 \mathrm{eV}$ [20], a much lower barrier height than our measured value or the value of Binari et al[1] would be expected. This finding can either be attributed to Fermi level pinning at the interface or to the presence of oxygen contamination at the interface, which is very likely since no Ultra High Vacuum system was used. After alloying the barrier height is determined by TiN which has a work function well below $4 \mathrm{eV}$ [21]. This would explain the ohmic contact behavior by the Schottky-Mott model. Another explanation would be the formation of a Ga-enriched interface and a low barrier height determined by the low work function of $\mathrm{Ga}(4.2 \mathrm{eV}[20])$.

The second classical method for ohmic contact formation involves a highly doped surface region beneath the contact. In the present case, an increased nitrogen vacancy concentration is anticipated at the interface after reaction, leading to an increased donor concentration.

Hence, the formation of an ohmic contact by alloyed Ti-contacts might be understood as superimposed effects of a low barrier height and an increased tunneling current caused by the nitrogen depletion at the GaN/TiN interface.

Besides these classical model explanations, the substrate itself and the pre-deposition treatment of the surface seem to play important roles in contact formation. Especially the influence of different etching procedures or heat treatment of the substrate is considerable. A possible cause for different results on pre-annealed substrates might be the incorporation of hydrogen into GaN during various processing steps and its removal upon annealing at higher temperatures [22]. Another explanation is that nitrogen depletion at the interface occurs at annealing temperatures around $900^{\circ}$ and leads to an increased donor density. This method was also used to obtain non-alloyed Ti/Al ohmic contacts by a premetallization anneal at $1120^{\circ} \mathrm{C}[23]$.

The discrepancies between authors who found Ti contacts to be ohmic as-deposited [3] [5] and studies who found rectifying behavior in the as-deposited state [this study] [24], can clearly be attributed to differences in substrate preparation. The former authors both used reactive ion etching which effectively removes oxide but also increases the defect density near the surface. In a recent study by transmission electron microscopy (TEM) [25] a thin TiN layer was observed even in as-deposited Ti contacts on RIE treated substrates.

All this findings support that the formation of a TiN layer is the dominant mechanism for the formation of Ti-based ohmic contacts. A thin TiN layer was identified at the GaN interface in reacted Ti/Al and Ti/Al/Ni/Au contacts[25] and thicker TiN layers seem to improve contact performance. The role of $\mathrm{Al}$ in these contacts, however, is still somewhat unclear. A possible explanation for the superior performance of Ti/Al contacts would be a phase other than TiN, which was not observed yet, is responsible for the contact behavior. We can speculate for example that small amounts of a ternary phase like $\mathrm{Ti}_{2} \mathrm{AIN}$ might form at the interface. Two ternary phases are stable at lower

temperatures in the Ti-Al-N ternary system [26]. The assumption of a ternary phase was also supported by RBS measurements of annealed Ti/Al contacts [27], where a mixture of all four participating elements was observed in the contact interface. For a complete view of this contact structure the more complicated phase equilibria in the quaternary Al-Ti-Ga-N system and the diffusion paths therein should be investigated.

\section{Conclusion}

TiN has been identified in diffusion couple experiments as a thermodynamically stable phase in contact with GaN. A layered $\mathrm{GaN} / \mathrm{TiN} / \mathrm{Ti}_{2} \mathrm{GaN} / \mathrm{Ti}_{3} \mathrm{Ga} / \mathrm{Ti}$ interface structure governs the electrical properties in alloyed Ti/GaN contacts. The electrical characterization suggests that TiN can be used as an ohmic contact material for GaN applications at high temperatures. In addition, a TiN layer could possibly serve as a diffusion barrier for additional metallizations. SinceTiN is a material which is already in use in microelectronics and can be deposited by sputtering as well as by MOVPE techniques, these findings might be useful for GaN process technology.

\section{Acknowledgments}

One of the authors, HC, wishes to acknowledge the German Research Council (Deutsche Forschungsgemeinschaft) for providing a research fellowship so that he could carry out this study. In addition, the authors would like to thank the National Science Foundation for partial financial support 
through Grant No. NSF-DMR-94-24478 and Prof. T.F. Kuech, University of Wisconsin for supplying the GaN substrate used in this study.

\section{References}

[1] S. C. Binari, H. B. Dietrich, G. Kelner, L. B. Rowland, K. Doverspike, D. K. Gaskill, Electron. Lett. 30, 909-911 (1994).

[2] M. E. Lin, Z. Ma, F. Y. Huang, Z. F. Fan, L. H. Allen, H. Morkoc, Appl. Phys. Lett. 64, 1003-1005 (1994).

[3] J. D. Guo , C. I. Lin, M. S. Feng , F. M. Pan , G. C. Chi , C. T. Lee , Appl. Phys. Lett. 68, 235-237 (1996).

[4] K.V. Vassilevski, M.G. Rastegaeva, A.I. Babanin, I.P. Nikitina, V.A. Dmitriev, MRS Internet J. Nitride Semicond. Res. 1, 38 (1996).

[5] Zhifang Fan, S. Noor Mohammad, Wook Kim, Ozgur Aktas, Andrei E. Botchkarev, Hadis Morkoc, Appl. Phys. Lett. 68, 1672-1674 (1996).

[6] SE Mohney, X Lin, J. Electron. Mater. 25, 811-818 (1996).

[7] JL Murray, Phase Diagrams of Binary Titanium Allloys (ASM International, Metals Park, Ohio, 1987) .

[8] M Poetschke, K Schubert, Z. Metall. 53, 474-488 (1962).

[9] W Jeitschko, H Nowotny, F Bebesowsky, Mon. Chem. 95, 178-179 (1964).

[10] J Klingbeil, R Schmid-Fetzer, J. Phase Equilibria 13, 522-531 (1992).

[11] FR de Boer, R Boom, WCM Mattens, AR Miedema, AK Niessen, Cohesion in Metals (North Holland, Amsterdam, 1988) .

[12] O Kubaschewski, CB Alcock, PJ Spencer, Materials Thermochemistry (Pergamon Press, Oxford, 1993) .

[13] P Villars, LD Calvert, Pearsons Handbook of Crystallographic Data for Intermettalic Phases (American Society of Metals, Metals Park,Ohio, 1985).

[14] W Kraus, G Nolze, PowderCell 1.8 (1996) .

[15] NR Perkins, MN Horton, RJ Matyi, ZZ Bandic, TC McGill, TF Kuechin 13th Int. Conference on CVD, , (Los Angeles, 1996) $\mathrm{x}$.

[16] GK Reeves, Sol. St. Electr. 23, 487-490 (1980).

[17] Lei Wang, M. I. Nathan , T-H. Lim , M. A. Khan, Q. Chen , Appl. Phys. Lett. 68, 1267-1269 (1996).

[18] J. S. Foresi, T. D. Moustakas , Appl. Phys. Lett. 62, 2859-2861 (1993).

[19] J. I. Pankove, H. Schade, Appl. Phys. Lett. 25, 53 (1974).

[20] HB Michaelson, J. Appl. Phys. 48, 4729-4732 (1977).

[21] VS Fomenko, GV Samsonov, Handbook of Thermionic Properties (Plenum Press, New York, 1966).

[22] SJ Pearton, RJ Shul, RG Wilson, F Ren, JM Zavada, CR Abernathy, CB Vartuli, JW Lee, JR Mileham, JD Mackenzie, J. Electron. Mater. 25, 845-849 (1996).

[23] LF Lester, JM Brown, JC Ramer, L Zhang, SD Hersee, JC Zolper, Appl. Phys. Lett. 69, 2737-2739 (1996). 
[24] AT Ping, MA Khan, I Adesida, J. Electron. Mater. 25, 819-824 (1996).

[25] S Ruvimov, Z Liliental-Weber, J Washburn, KJ Duxstad, EE Haller, Z-F Fan, SN Mohammad, W Kim, AE Botchkarev, H Morkoc, Appl. Phys. Lett. 69, 1556-1558 (1996).

[26] JC Schuster, J Bauer, J. Sol. St. Chem. 53, 260-265 (1984).

[27] QZ Liu, SS Lau, N Perkins, M Horton, TF Kuech, unpublished (1996).
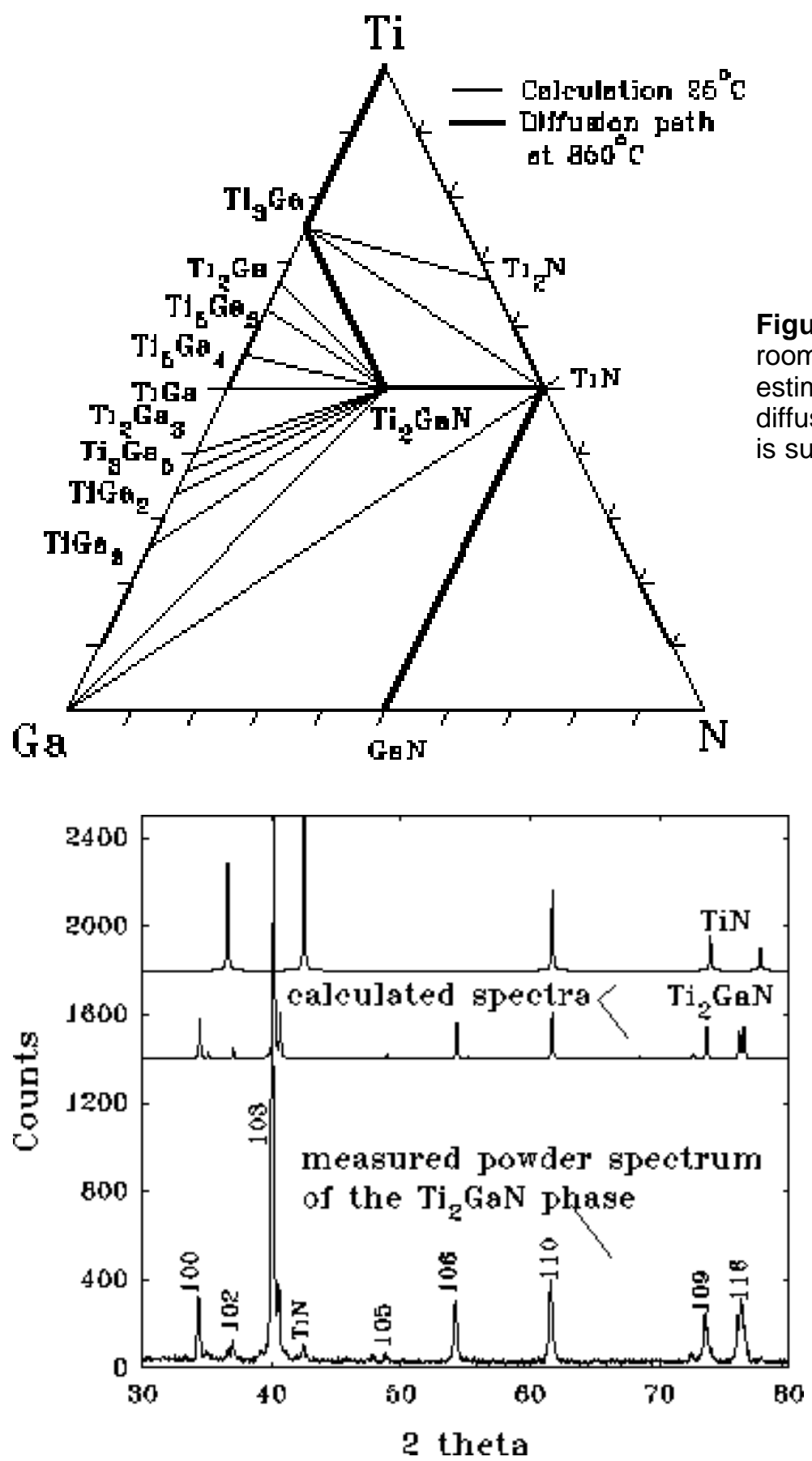

Figure 1. Isothermal section of the Ti-Ga-N system at room temperature, approximately calculated with partly estimated thermodynamic values. The observed diffusion path in diffusion couples, annealed at $850^{\circ} \mathrm{C}$, is superimposed.
Figure 2. The ternary $\mathrm{Ti}_{2} \mathrm{GaN}$ phase, produced by solid state reaction from $\mathrm{TiN}, \mathrm{Ga}$ and $\mathrm{Ti}$, was confirmed by XRD. The calculated $x$-ray spectra of $\mathrm{Ti}_{2} \mathrm{GaN}$ and TiN are also given for comparison. 


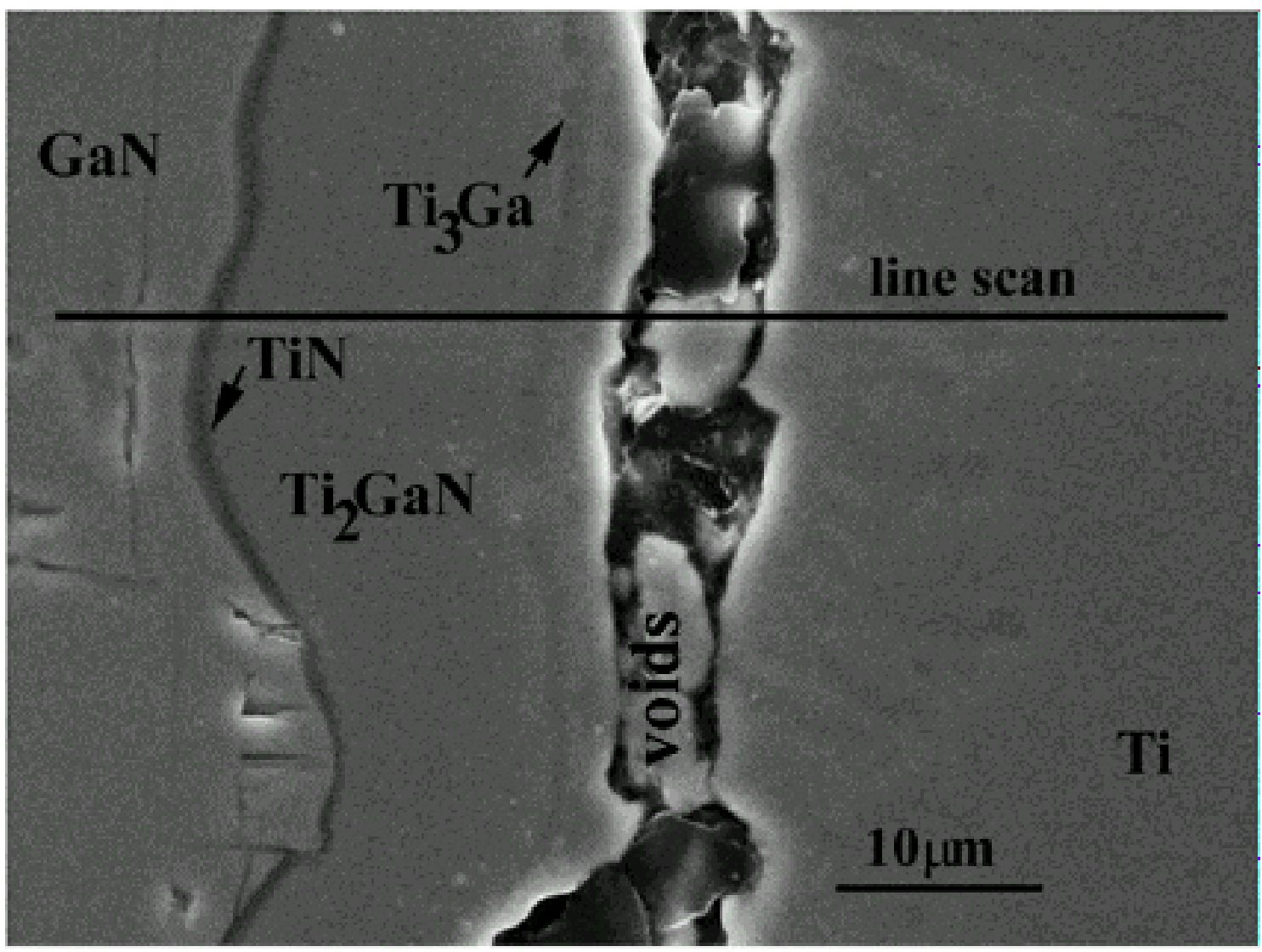

Figure 3a. Section across the interface of a massive Ti/GaN diffusion couple annealed at $850^{\circ} \mathrm{C} / 2 \mathrm{~d}$. A GaN/TiN/Ti ${ }_{2} \mathrm{GaN} / \mathrm{Ti}_{3} \mathrm{Ga} / \mathrm{Ti}$ diffusion path was identified by EDX and XRD.

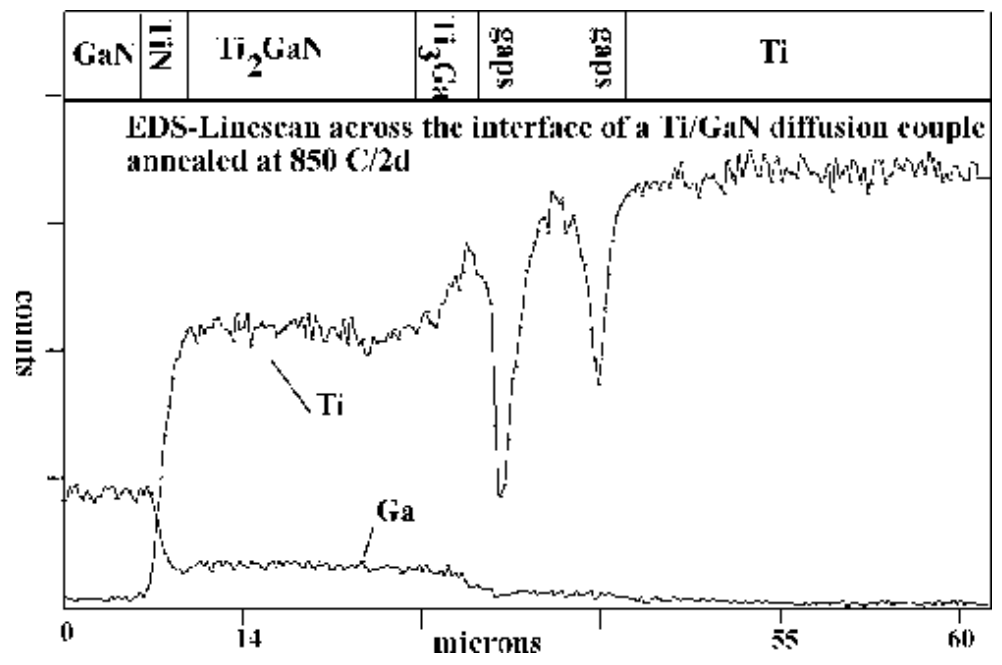

Figure 3b. Corresponding EDX line scan across the interface 


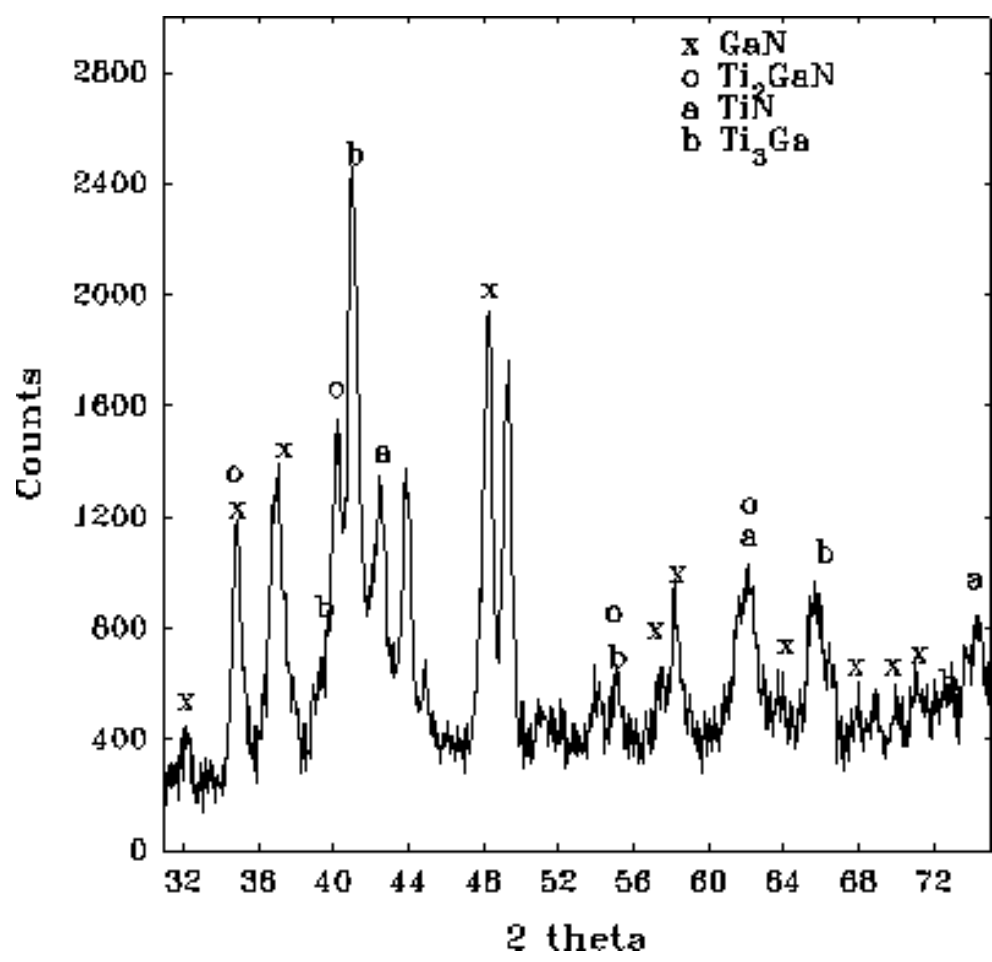

Figure 4. XRD spectrum of a thin film Ti/GaN diffusion couple, annealed at $850^{\circ} \mathrm{C} / 1 \mathrm{~d}$, compared with spectra of reaction phases.

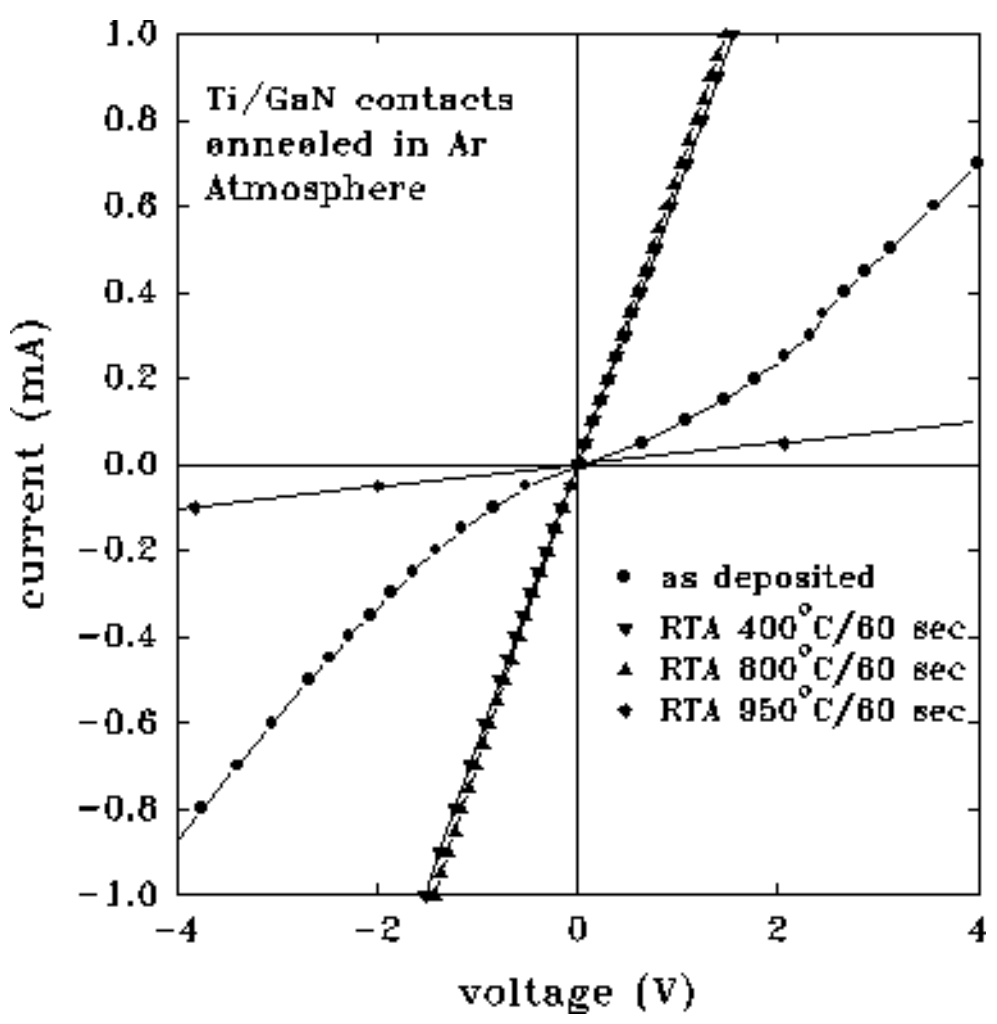

Figure 5. Current-voltage characteristics of Ti/GaN contacts annealed at various temperatures in Argon. The curves for temperatures between 400 and $800^{\circ} \mathrm{C}$ are omitted for clarity. 


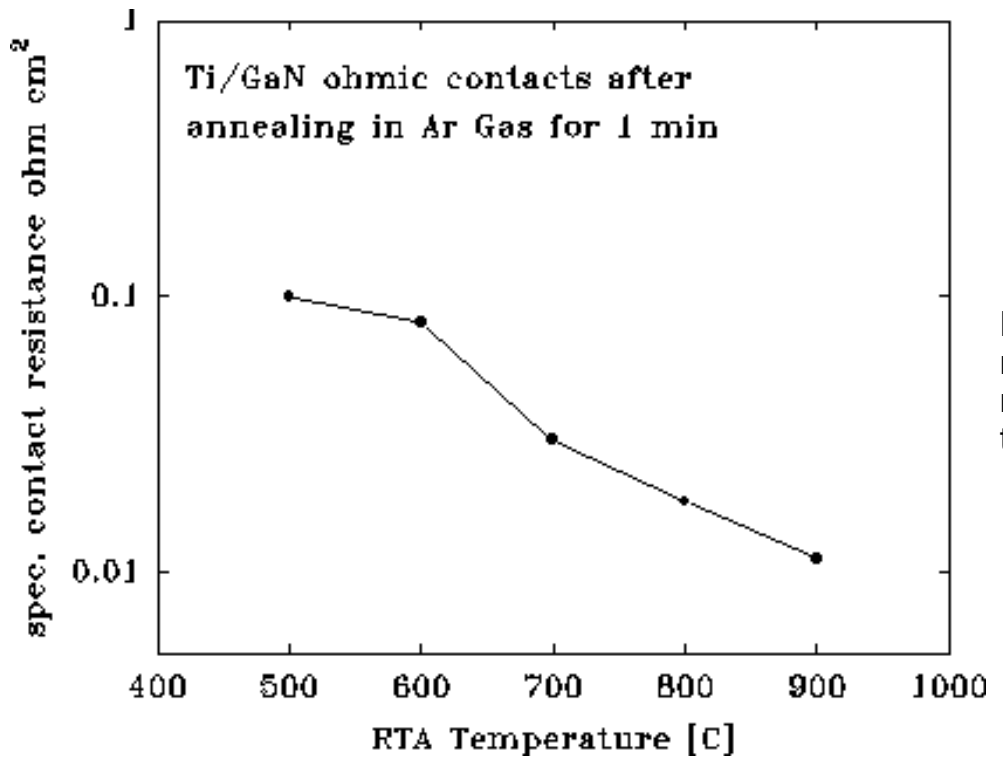

Figure 6. Specific contact resistance of $\mathrm{Ti} / \mathrm{GaN}$, measured by the circular transmission line method, as a function of RTA annealing temperature.

(C) 1997 The Materials Research Society

\begin{tabular}{|l|l|l|l|l}
\hline $\mathrm{M}$ & $\mathrm{R}$ & $\mathrm{S}$ & Internet Journal of & Nitride Semiconductor Research \\
\hline
\end{tabular}

